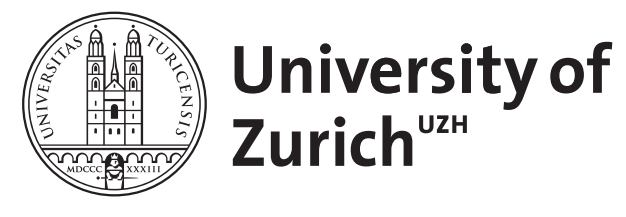

\title{
Invasive Techniken in der Notfallmedizin
}

\author{
Mutzbauer, T S ; Keul, W ; Bernhard, M ; Völkl, A ; Gries, A
}

\begin{abstract}
Die Koniotomie im Notarztdienst ist eine sehr invasive Methode zur Atemwegssicherung. Sie ist aber unabdingbar, wenn andere weniger invasive Verfahren der Atemwegssicherung misslingen oder sich situationsabhängig als nicht zweckmäßig erweisen. Für die Durchführung wurden verschiedene Verfahren beschrieben, die in anatomisch-chirurgische Präpariertechniken und Punktionsverfahren unterteilt werden können. Das im jeweiligen Notarztsystem bevorzugte Verfahren orientiert sich meist an abteilungsüblichen Standards, die vom klinischen Vorgehen abgeleitet werden. Ausbildungs- und Trainingsmöglichkeiten für die einzelnen Verfahren bestehen in der Intensivmedizin, am Leichenpräparat und am Phantom. In dieser Arbeit werden verschiedene Methoden der Koniotomie besprochen sowie eine anatomische Präpariertechnik und 2Punktionsverfahren im Detail dargestellt
\end{abstract}

DOI: https://doi.org/10.1007/s00101-004-0768-z

Posted at the Zurich Open Repository and Archive, University of Zurich

ZORA URL: https://doi.org/10.5167/uzh-155820

Journal Article

Published Version

Originally published at:

Mutzbauer, T S; Keul, W; Bernhard, M; Völkl, A; Gries, A (2005). Invasive Techniken in der Notfallmedizin. Der Anästhesist, 54(2):145-154.

DOI: https://doi.org/10.1007/s00101-004-0768-z 
Anaesthesist $2005 \cdot 54: 145-154$ DOI 10.1007/s00101-004-0768-z

Online publiziert: 15. Januar 2005

(c) Springer Medizin Verlag 2005

T. S. Mutzbauer ${ }^{1} \cdot$ W. Keul ${ }^{2} \cdot$ M. Bernhard ${ }^{2} \cdot$ A. Völk| $\left.\right|^{3}$ A. Gries ${ }^{2,4}$

${ }^{1}$ Klinik für Kiefer- und Gesichtschirurgie, Universitätsspital, Zürich, Schweiz

${ }^{2}$ Klinik für Anaesthesiologie, Bereich Notfallmedizin, Ruprecht-Karls-Universität, Heidelberg

${ }^{3}$ Anatomisches Institut II, Ruprecht-Karls-Universität, Heidelberg

${ }^{4}$ Deutsche Rettungsflugwacht, Luftrettungszentrum „Christoph 53", Mannheim

\section{Invasive Techniken in der Notfallmedizin}

\section{Koniotomie im Notarztdienst}

ereits im 16. Jh. wurde der chirurgische Zugang zum Atemweg als lebensrettend beschrieben. Im 19. Jh. konnten zahlreiche an Diphtherie erkrankte Patienten durch eine Tracheotomie, die als Notfallmaßnahme erkannt worden war, vor dem drohendem Erstickungstod bewahrt werden [15]. Im Jahr 1957 wurde das Lig. cricothyreoideum als die am oberflächlichsten gelegene Stelle des Atemwegs unterhalb der Glottisebene und somit als die ideale Lokalisation für einen notfallmäßigen chirurgischen Atemwegszugang vorgeschlagen $[5,10]$. Mittlerweile hat die Koniotomie in den Leitlinien und Empfehlungen internationaler Fachgesellschaften zum Management des schwierigen Atemwegs einen festen Platz $[6,19]$. Aufgrund ihrer vergleichsweise hohen Invasivität wird ihre Anwendung jedoch nur dann empfohlen, wenn andere Maßnahmen fehlschlagen oder nicht ausreichend schnell durchgeführt werden können.

Im Notarztdienst sind allerdings einige klinische Methoden kaum realisierbar. Insbesondere die „Rückwärtsstrategie“, also die Möglichkeit einen Patienten bei fehlgeschlagener Atemwegskontrolle nach Narkoseeinleitung wieder aufwachen zu lassen, ist häufig nicht praktikabel oder undurchführbar. Da viele Patienten bereits bewusstlos oder aufgrund lebensbedrohlicher Erkrankung bzw. Verletzung beat- mungspflichtig sind, ist oft auch ein Zeitverlust bei der Sicherung der Atemwege nicht akzeptabel. Darüber hinaus sind einige in der Routineanästhesie gebräuchlichen Hilfsmittel zur Atemwegssicherung im Notarztdienst in aller Regel nicht verfügbar. Dies gilt derzeit insbesondere für die flexible Fiberoptik, die zumindest im deutschsprachigen Raum unter klinischen Bedingungen einen hohen Stellenwert im Rahmen des Atemwegsmanagements einnimmt. Zwar wurde ihr Einsatz auch im Rettungsdienst bereits publiziert [27]; eine weite Verbreitung hat sie aufgrund ihres hohen Anschaffungspreises und der für dieses Verfahren notwendigen großen klinischen Erfahrung bisher jedoch nicht erlangt. Aus den genannten Gründen folgt, dass die Koniotomie gerade für den Notarztdienst eine besonders hohe Bedeutung besitzt.

\section{Indikationen}

In Anlehnung an die oben genannten Leitlinien können für den Notarztdienst grundsätzlich 2 Szenarien unterschieden werden, bei denen die Indikation zur Koniotomie gestellt wird $[6,19]$ :

\section{(1) Maskenbeatmung und Intubation miss- lingen, die Oxygenierung des Patienten ist nicht gewährleistet.}

In dieser als „cannot-ventilate-cannot-intubate" bezeichneten Situation kann als zusätzliche Alternative vor der Koniotomie noch die Beatmung über eine supraglottische Atemhilfe (z. B. Larynxmaske, Larynxtubus, „Combitube“, „Easytube“) versucht werden. Misslingt deren Anwendung ebenfalls oder stehen diese Hilfsmit-

Tabelle 1

Hindernisse im Bereich von

Mund/Rachen und Kehlkopf bzw.

zum intubationsgerechten

Zugang

Zungenschwellung (z. B. allergisch durch Insektenstich, ACE-Hemmer, hereditäres Angioödem)

Verbrennung von Gesicht und Hals mit Weichteilschwellung, Inhalationstauma mit Schleimhautschwellung Blutung in Mund/Rachen bei Mittelgesichtstrauma oder Schädelbasisfraktur

Bolusgeschehen

Verletzung des Kehlkopfskeletts (laryngeales Trauma)

Eingeklemmter Patient

ACE Angiotensinkonversionsenzym 
Tabelle 2

Entscheidungshilfen

Welche Alternativmethoden stehen zur Verfügung?

Mit welcher Methode hat der Notarzt am meisten Erfahrung?

Wie hoch ist die Aspirationsgefahr einzuschätzen (Anamnese)?

Wie lange dauert der Transport voraussichtlich (Stadt/Land)?

Welche Interventionsmöglichkeiten bestehen während des Transports (Rettungswagen/Hubschrauber)?

Tabelle 3

Koniotomieinstrumentarium für den mobilen Einsatz

Metzenbaum-Präparierschere

Killian-Spekulum, lang

2 Halsted-Mosquito-Klemmen, gebogen, scharf

Nadelhalter

Pinzette, chirurgisch

Transparentes steriles Lochtuch

Mallinckrodt Safety-Flex ${ }^{\circledR}$ Tubus,

ID 6,5-5,0 mm

(Drahtspiraltubus mit „low pressure cuff“)

Führungsstab

Skalpell Nr. 11

Atraumatisches Nahtmaterial 2-0, geflochten

Sterile Kompressen

Chirurgischer Sauger

(mit Verlängerungsschlauch

zum Anschluss an Absaugpumpe)

tel nicht zur Verfügung, um eine Hypoxie abzuwenden, muss die Koniotomie sofort durchgeführt werden. Die Notwendigkeit einer Koniotomie ergibt sich v. a. dann, wenn weiche oder harte Hindernisse den Kehlkopf oder die oberen Atemwege verschließen und ein Atemwegszugang ,von oben“ unmöglich ist (•Tabelle 1$)$.

(2) Die endotracheale Intubation misslingt, eine Oxygenierung über Gesichtmaske oder supraglottische Atemhilfen ist zwar möglich, erscheint aber für den weiteren präklinischen Verlauf als nicht ausreichend.
Folgende Überlegungen spielen hier eine Rolle:

- Bei Notfallpatienten muss immer mit fehlender Nüchternheit und daraus resultierender Aspirationsgefahr gerechnet werden. Nicht zuletzt deshalb gilt die endotracheale Intubation als ,golden standard“ der Atemwegssicherung im Notfall. Im Vergleich dazu erhöht sich bei der Maskenbeatmung die Aspirationsgefahr mit zunehmender Anwendungsdauer [28]. Die Larynxmaske verringert zwar die Aspirationsgefahr im Vergleich zur Gesichtsmaske; einen sicheren Aspirationsschutz stellt sie jedoch ebenfalls nicht dar [8]. Lediglich Combitube und Easytube scheinen durch die Blockung im Ösophagus und die Möglichkeit der Ableitung von Mageninhalt durch das ösophageale Lumen nach außen eine Aspiration von Mageninhalt am ehesten zu vermeiden [1]. Bei schweren Blutungen in den Rachenraum, z. B. aufgrund eines Mittelgesichtstraumas oder einer Schädelbasisfraktur, können supraglottische Atemhilfen eine Aspiration von Blut nicht sicher verhindern.

- Eine Vorwärtsstrategie in Richtung Koniotomie kann auch dann indiziert sein, wenn bei einer Schwellung der oberen Atemwege oder der Halsweichteile, z. B. bei Verbrennungen oder im Rahmen von allergischen Reaktionen, eine Oxygenierung über die Gesichtmaske oder supraglottische Atemhilfen gelingt, aber mit einer zunehmenden schwellungsbedingten Obstruktion der Atemwege und dadurch mit einem sekundären Verlust der Beatmungsmöglichkeit gerechnet werden muss.

Die Beantwortung der Frage, ob die Indikation für eine Koniotomie trotz aktuell möglicher Oxygenierung gestellt werden muss, hängt also von zahlreichen Einzelfaktoren ab, die individuell abgewogen werden müssen ( $\bullet$ Tabelle 2 ).

\section{Inzidenz in der präklinischen Notfallmedizin}

Die Häufigkeit einer Koniotomie im Rettungsdienst schwankt stark in Abhängig- keit vom Anwenderkollektiv. An einem US-amerikanischen Traumazentrum mit angeschlossenem Luftrettungsdienst wurden in einem 5-Jahres-Zeitraum 2730 Intubationen durchgeführt, davon etwa 90\% im Schockraum und 10\% präklinisch. Die präklinisch im Luftrettungsdienst tätigen und in einer chirurgischen Koniotomietechnik („,Rapid-four-stepTechnik") ausgebildeten "flight nurses“ führten mit 10,9\% deutlich häufiger Koniotomien durch als Ärzte im Schockraum $(1,1 \%)[3,9]$. In einer älteren Untersuchung an einem deutschen unfallchirurgisch besetzten Luftrettungszentrum wird die Inzidenz der Koniotomie mit 2,4\% angegeben [17]. Demgegenüber wurde im eigenen, ausschließlich mit Anästhesisten besetzten Luftrettungsdienst mit jährlich rund 1000, zur Hälfte beatmeten und überwiegend traumatologischen Notfallpatienten, die Indikation zur Koniotomie nur in o, $1 \%$ der Fälle gestellt [24].

Der Vergleich dieser Zahlen zeigt, dass die Koniotomie zwar durchaus eine Indikation in der Notfallmedizin hat, ihr Stellenwert jedoch erheblich von der Routine des eingesetzten Personals mit den Methoden der konventionellen Atemwegssicherung abhängt.

\section{Anatomische Grundlagen}

Die Lokalisation zur Anlage einer Koniotomie befindet sich in der unter der Haut tastbaren Lücke zwischen Schild- und Ringknorpel. Prominenteste Struktur ist der Schildknorpel (Cartilago thyreoidea) mit dem Adamsapfel (Prominentia laryngea). Zwischen Schildknorpel und Ringknorpel spannt sich das Lig. cricothyreoideum (Synonym: Lig. conicum) aus die Zielstruktur der Koniotomie. Wesentliche Strukturen, die sich in unmittelbarer Nähe dieses Zugangsweges befinden und daher im Rahmen der Koniotomie verletzt werden können, sind die Schilddrüse (Glandula thyreoidea, ggf. mit akzessorischem Lobus pyramidalis) mit ihren zugehörigen Blutgefäßen (Vasa thyreoidea superiores et inferiores), die großen Halsgefäße (A. carotis communis, $\mathrm{Vv}$. jugulares interna et externa), sowie der unmittelbar hinter der Trachea liegende Ösophagus (• Abb. 1). 


\section{Techniken und Material}

Eine Reihe verschiedener Präpariertechniken und Punktionsverfahren wurde beschrieben:

1. Anatomisch-chirurgische Präpariertechniken:

a. konventionelle Präparation,

b. Rapid-four-step-Technik [9].

2. Punktionsverfahren:

a. Stahlinnenkanülentechnik:

Jet-Ventilationskatheter nach

Ravussin [26],

behelfsmäßig mit großlumiger

Venenverweilkanüle,

Koniotomiekanülen mit großem Innendurchmesser, z. B. Quicktrach $4 \mathrm{~mm}[16]$,

b. Seldinger-Technik, z. B. MelkerKoniotomie-Set mit blockbarer Kanüle [11, 14].

Unabhängig davon, welche der oben genannten Techniken eingesetzt werden, müssen folgende Anforderungen erfüllt sein: Das Instrumentarium muss unmittelbar zur Verfügung stehen, leicht zu handhaben und mit einem Standardkonnektor zum Anschluss der üblichen Beatmungseinheiten versehen sein. Das Verletzungsrisiko für benachbarte Strukturen sollte so gering wie möglich sein [16].

Eine Kopfüberstreckung erleichtert die Durchführung aller oben genannten Koniotomietechniken. Beim Training am Leichenpräparat gelang jedoch regelhaft allenfalls eine moderate Überstreckung des Kopfes, ohne dass sich Anhaltspunkte ergaben, dass die Maßnahme dadurch erschwert oder verzögert wurde [23, 24]. Die Bewegungen, die sich aus der Durchführung einer Koniotomie bei einem $\mathrm{Pa}$ tienten mit einer bestehenden Halswirbelsäulenverletzung ergeben und damit die potenzielle Gefahr, die Folgen einer derartigen Verletzung zu aggravieren, dürften bei moderater Kopfüberstreckung klinisch kaum relevant sein [18] - jedenfalls hat nach Ansicht der Autoren die Atemwegssicherung Priorität.

Im Folgenden werden ein chirurgischanatomisches Verfahren, ein Punktionsverfahren mit Stahlinnenkanülentechnik und eine Seldinger-Technik im Detail beschrieben.

Anaesthesist $2005 \cdot 54: 145-154$

DOI 10.1007/s00101-004-0768-z

c) Springer Medizin Verlag 2005

\section{T. S. Mutzbauer · W. Keul • M. Bernhard · A. Völkl · A. Gries}

\section{Invasive Techniken in der Notfallmedizin. IV. Koniotomie im Notarztdienst}

Zusammenfassung

Die Koniotomie im Notarztdienst ist eine sehr invasive Methode zur Atemwegssicherung. Sie ist aber unabdingbar, wenn andere weniger invasive Verfahren der Atemwegssicherung misslingen oder sich situationsabhängig als nicht zweckmäßig erweisen. Für die Durchführung wurden verschiedene Verfahren beschrieben, die in anatomisch-chirurgische Präpariertechniken und Punktionsverfahren unterteilt werden können. Das im jeweiligen Notarztsystem bevorzugte Verfahren orientiert sich meist an abteilungsüblichen Standards, die vom klinischen Vorgehen abgeleitet werden. Ausbildungs- und Trainingsmöglichkeiten für die einzelnen Verfahren bestehen in der Intensivmedizin, am Leichenpräparat und am Phantom. In dieser Arbeit werden verschiedene Methoden der Koniotomie besprochen sowie eine anatomische Präpariertechnik und 2 Punktionsverfahren im Detail dargestellt.

Schlüsselwörter

Koniotomie $\cdot$ Notarztdienst .

Invasive Methoden · Atemwegssicherung

\section{Invasive techniques in emergency medicine. IV. Cricothyrotomy in emergency situations}

\section{Abstract}

Cricothyrotomy is a very invasive technique to secure the airway in an emergency but is irreplacable when less invasive techniques fail or cannot be instigated under the prevailing circumstances. Various techniques have been reported which can be subdivided into anatomical-surgical preparation or puncture techniques. The preferred strategy is mostly oriented towards the departmental standard procedure which will be decided by the clinical situation. Training for each procedure can be carried out in intensive care departments, and using autopsy material or a manekin. Various methods of cricothyrotomy will be discussed here, and additionally an anatomical preparation and two puncture techniques will be demonstrated in detail.

\section{Keywords}

Cricothyrotomy - Emergency service . Invasive techniques - Airway management 


\section{Notfallmedizin}
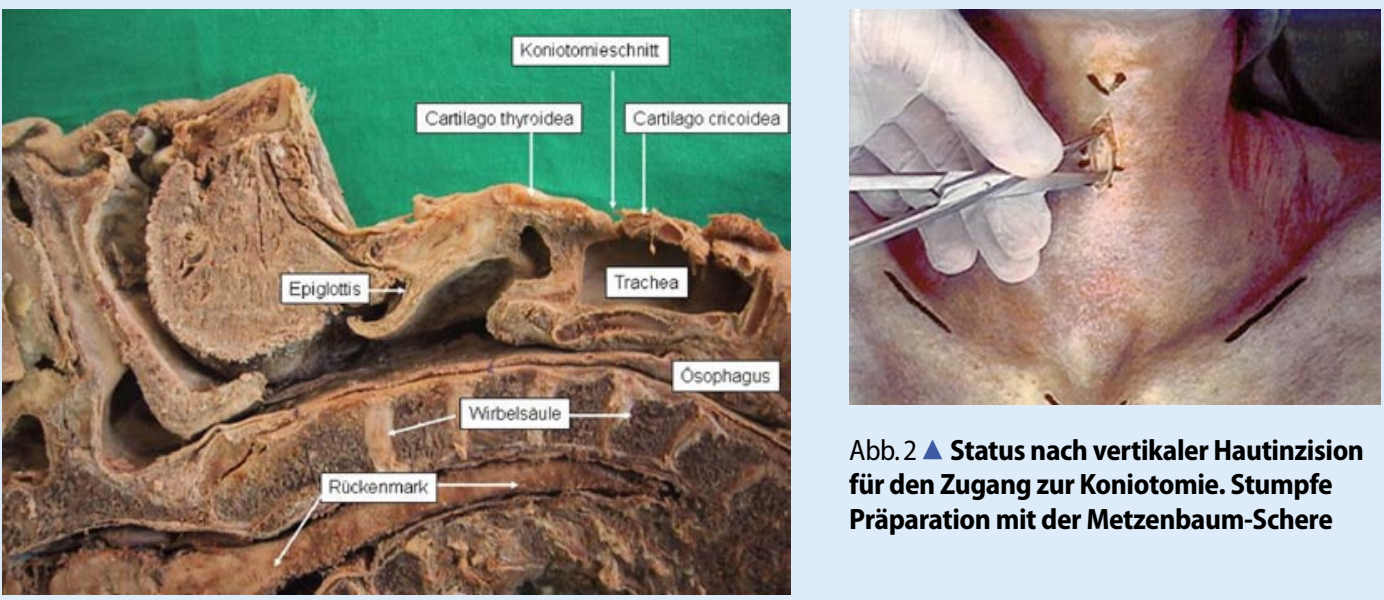

Abb. 1 Anatomische Darstellung der für die Koniotomie relevanten anatomischen Strukturen

im Sagittalschnitt am fixierten Leichenpräparat

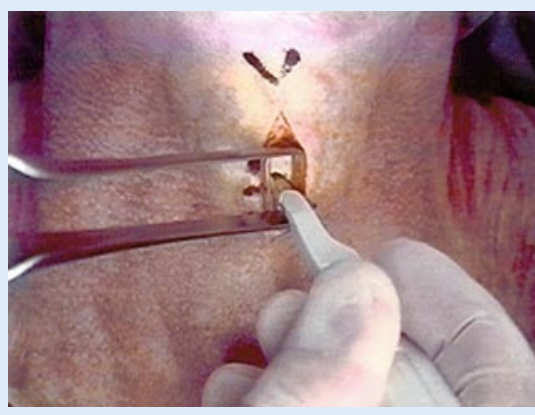

Abb. $3 \triangle$ Querinzision der Membrana cricothyreoidea mit dem Skalpell (eigentliche Koniotomie)

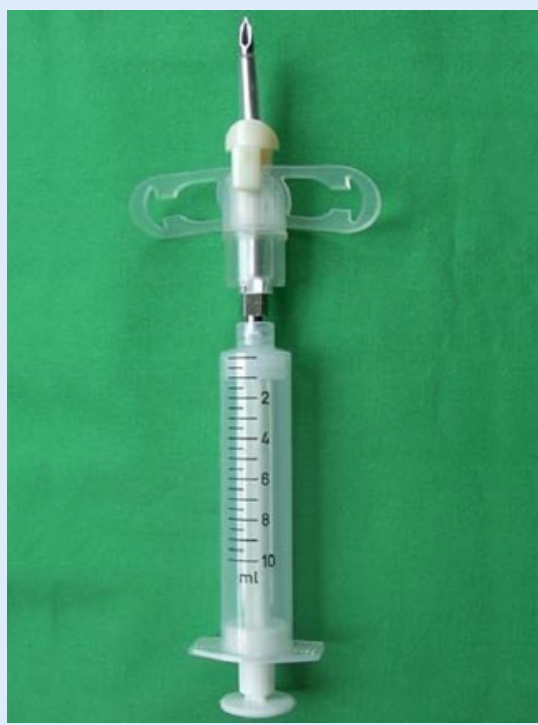

Abb.6 $\Delta$ Quicktrach nach Entfernung aus der sterilen Umverpackung

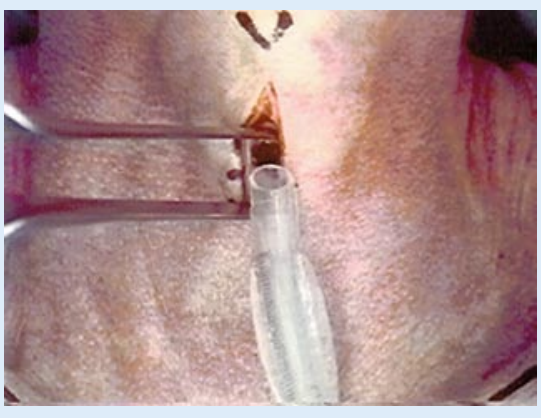

Abb. $4 \Delta$ Vor Einführen des Endotrachealtubus unter Zuhilfenahme des Killian-Spekulums nach Koniotomie. Tubusspitze zeigt nach noch nach kranial. Im Verlauf der Tubusinsertion wird dieser zunächst in der Sagittalebene um $90^{\circ}$, dann um $180^{\circ}$ gedreht und dann nach kaudal vorgeschoben. Es ist auch möglich, den Tubus innen mit einem Führungskatheter zu armieren. Dies erleichtert die Einführung und verhindert ein Abknicken der Tubusspitze nach kranial

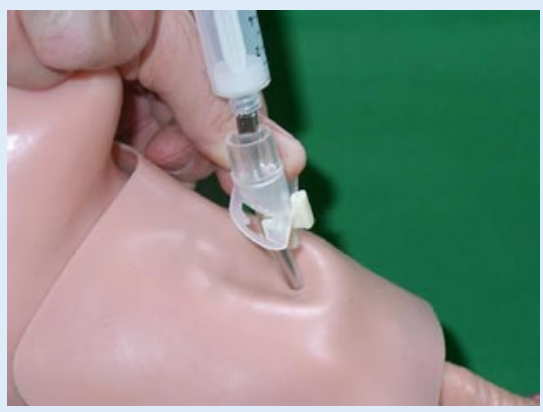

Abb.7 $\triangle$ Einstechen der Quicktrach-Kanüle durch die Haut über dem Lig. cricothyreoideum. Dabei wird ein Kanülenflügel gesichert und dem Quicktrach damit eine zusätzliche Richtungsstabilität gegeben
Abb. $2 \triangle$ Status nach vertikaler Hautinzision für den Zugang zur Koniotomie. Stumpfe Präparation mit der Metzenbaum-Schere

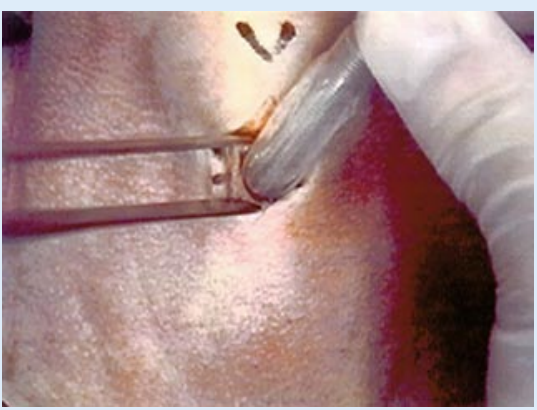

Abb. 5 Einführen des Endotrachealtubus nach Koniotomie. Wichtig ist, dass das Killian-Spekulum dabei leicht zurückgezogen wird, da die Branchen nicht gleichzeitig mit dem Tubus zwischen Ring- und Schildknorpel Platz finden

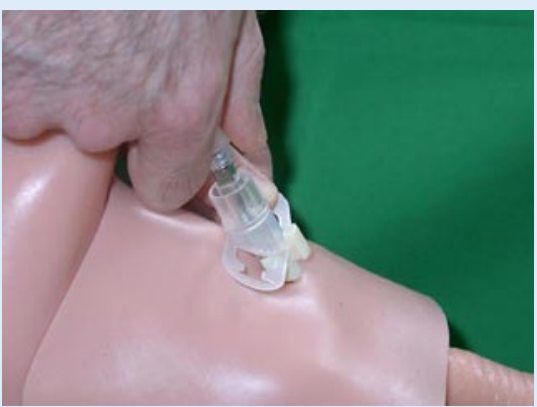

Abb. $8 \triangle$ Die vom Hersteller mit dem Quicktrach vorkonnektierte 10-ml-Spritze muss bei der Punktionskoniotomie fest in die Hand genommen werden 
Anatomisch-chirurgische

Präpariertechniken

\section{Konventionelle Präpariertechnik}

Im Folgenden wird die Technik mit vorgepacktem Instrumentarium (• Tabelle 3 ) beschrieben:

- Der Notarzt steht oder kniet (als Rechtshänder) rechts vom Patienten.

- Palpatorische Lokalisation des Schildund Ringknorpels mit dem Zeigefinger der Arbeitshand und Fixieren des Schildknorpels mit Daumen und Zeigefinger der anderen Hand in Form eines nach kaudal offenen $\mathrm{V}$ [25].

- Längsinzision der Haut in der Mittellinie (Skalpell Nr. 11), beginnend über der Mitte des Schildknorpels und endend über dem Ringknorpel (Schnittlänge max. $3,5 \mathrm{~cm}$ ).

- Stumpfes Spreizen des Unterhautgewebes mit der Präparierschere, Spaltung der Fascia praetrachealis, Auseinanderdrängen der infrahyoidalen Muskulatur und evtl. vorliegender Strukturen (Lobus pyramidalis oder Gefäße;

\section{- Abb. 2)}

- Querinzision der Membrana cricothyreoidea (Lig. conicum) und der angrenzenden Trachealschleimhaut mit dem Skalpell unter Zuhilfenahme des Killian-Spekulums (• Abb. 3).

- Darstellung der Öffnung in der Membrana cricothyreoidea mit dem Killian-Spekulum, ohne dabei die beiden Knorpel visierartig auseinander zu drängen.

- Einführen des mit einem Führungsmandrin (Spitze sollte leicht über die Tubusspitze herausstehen) armierten Endotrachealtubus (• Abb. 4; hier ohne Mandrin dargestellt). Der Tubus sollte eine abgeschrägte Spitze aufweisen; damit lässt sich das allmähliche Auseinanderdrängen der Gewebsstrukturen beim Einführen des Tubus unter gleichzeitigem Zurückziehen des Killian-Spekulums erzielen. Außerdem gleitet ein abgeschrägter Tubus nach Zurückziehen des Mandrins leichter an der Trachealhinterwand entlang, so dass ein Abknicken nach kranial verhindert wird.

- Zurückziehen des Killian-Spekulums während der Tubusinsertion, da die geschaffene Öffnung nicht gleichzeitig Tubus und Spekulumbranchen aufnehmen kann (• Abb.5).

- Blocken des Tubus-Cuffs und Auskultationskontrolle.

- Sicherung des Tubus mit Nahtmaterial im Hautniveau; ggf. kann auch eine Mullbinde verwendet werden.

\section{Rapid-four-step-Technik}

Die Rapid-four-step-Technik stellt eine Modifikation dieses Vorgehens dar: Der Notarzt steht oder kniet am Kopfende des Patienten und führt eine Querinzision von Haut und Lig. cricothyreoideum durch. Dann wird der Ringknorpel mit einem speziellen Trachealhaken gefasst und zusammen mit dem kaudal davon gelegenen Trachealanteil nach ventral und kaudal gezogen, so dass der Endotrachealtubus jetzt unterhalb des Hakens in die Trachea geschoben werden kann [9].

\section{Punktionsverfahren}

\section{Stahlinnenkanülentechnik}

Die Beschreibung erfolgt am Beispiel des Quicktrach-Punktions-Sets:

- Der Notarzt steht oder kniet hinter dem Kopf des Patienten.

- Palpatorische Lokalisation der Lücke zwischen Schild- und Ringknorpel.

- Punktion der Haut und der Membrana cricothyreoidea mit der Quicktrach-Kanüle (• Abb. 6). Die vom Hersteller (VBM, Sulz a.N.) vorkonnektierte 10-ml-Spritze muss bei der Punktionskoniotomie fest in die Hand genommen werden, während der Kanülenflügel mit der anderen Hand gesichert und dem Quicktrach damit eine zusätzliche Richtungsstabilität gegeben wird (• Abb. 7 und 8 ). Verschiedene Anwender empfehlen, die Spritze vorher mit 2-3 ml Kochsalzlösung zu befüllen, so dass die erfolgreiche Punktion der Trachea durch die Aspiration von Luftblasen erkannt werden kann. Die aufgesetzte Stoppvorrichtung soll dabei eine Läsion der Tracheahinterwand bzw. eine Perforation zum Ösophagus verhindern. Eine Stichinzision durch die Haut vor der eigentlichen Punktion wird zwar vom Hersteller nicht vorge- geben, kann aber den erforderlichen Kraftaufwand reduzieren.

- Luftaspiration über die Spritze

(• Abb. 9).

- Entfernen der Stoppvorrichtung

(• Abb. 10).

- Vorschieben des Kunststoffkatheters über die Punktionskanüle unter Abstützung mit der Hand, die die Spritze fixiert, (• Abb. 11; am Kinn $(A)$ ) und Auskultationskontrolle.

- Sicherung des Katheters mit einer Haltenaht.

\section{Seldinger-Technik}

Mit dem Melker-Koniotomie-Set (Cook, Mönchengladbach) mit blockbarer Kanüle $(5 \mathrm{~mm} \mathrm{ID})$ wird wie folgt vorgegangen (- Abb. 12) [21]:

- Der Notarzt steht oder kniet hinter dem Kopf des Patienten.

- Palpatorische Lokalisation der Lücke zwischen Schild- und Ringknorpel.

- Punktion der Haut und der Membrana cricothyreoidea mit der Punktionskanüle und aufgesetzter Spritze.

- Luftaspiration über die Spritze. (Auch hier kann die Spritze vorher mit 2$3 \mathrm{ml}$ Kochsalzlösung befüllt werden, um die erfolgreiche Punktion der Trachea durch die Aspiration von Luftblasen erkennen zu können.)

- Absetzen der Spritze, Einführen des Führungsdrahts und Herausziehen der Punktionsnadel.

- Hautinzision beidseits neben dem Führungsdraht, jeweils $0,5-1 \mathrm{~cm}$.

- Vorschieben von Dilatator und Koniotomiekanüle über den Führungsdraht in die Trachea (• Abb. 13).

- Entfernen des Dilatators, Blocken des Cuffs und Auskultationskontrolle (- Abb. 13; kleines Bild: Lage in situ). - Sicherung der Koniotomiekanüle mit einer Haltenaht.

\section{Vergleich der verschiedenen Verfahren}

Folgende Kriterien lassen eine Bewertung der verschiedenen Methoden im Vergleich zu:

- Zeitbedarf bis zur Oxygenierung,

- Qualität der Ventilation, 


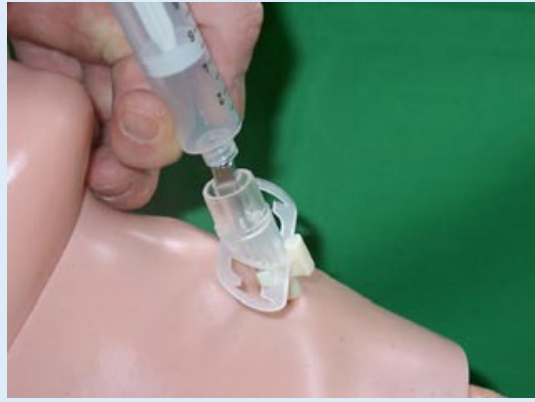

Abb. $9 \Delta$ Aspiration von Luft über die vorkonnektierte 10-ml-Spritze

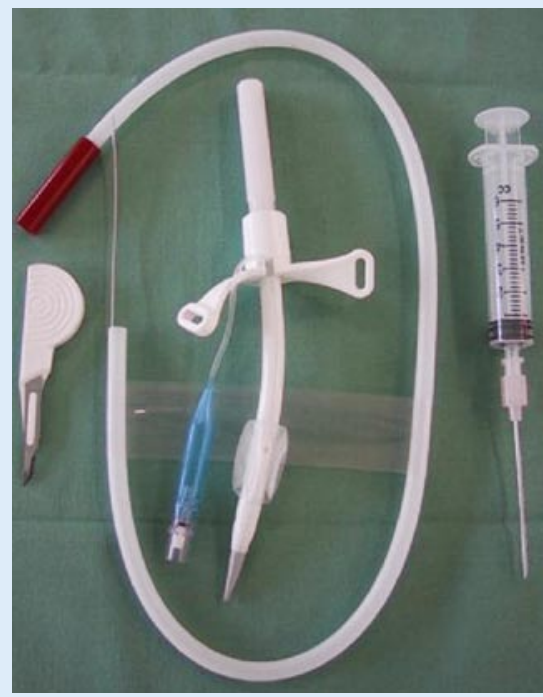

Abb. $12 \Delta$ Melker-Koniotomie-Set mit Punktionsbesteck, Führungsdraht, Dilatator und blockbarer Kanüle

- Aspirationsschutz,

- Komplikationsrate.

\section{Zeitbedarf}

Der Zeitbedarf zwischen einer Punktions(13-G-Jetventilationskatheter) und der oben beschriebenen chirurgisch-anatomischen Koniotomietechnik wurde von den Autoren an unfixierten Leichen untersucht [24]. Dabei konnten die Anwender (Weiterbildungsassistenten der Anästhesiologie im 4. Weiterbildungsjahr) in durchschnittlich 25 s (Bereich 10-36 s) die Punktion des Lig. conicum vornehmen, während die chirurgische Technik 73 s (53-255 s) in Anspruch nahm. Eine andere Anwendergruppe, bestehend aus Medizinstudenten und weniger erfahrenen Weiterbildungsassis-

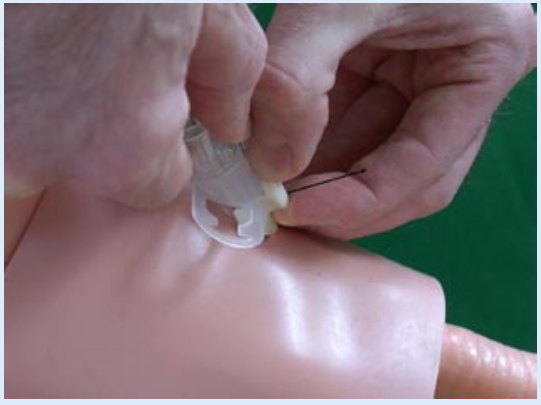

Abb. $10 \triangle$ Entfernen der Sicherheits-StoppVorrichtung

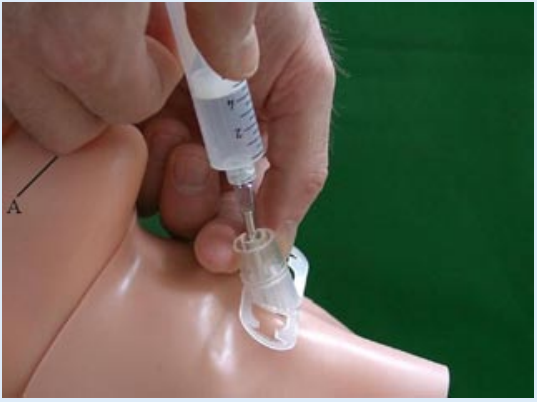

Abb. $11 \Delta$ Weiteres Vorschieben der Kunststoffaußenkanüle des Quicktrach unter Abstützung $(A)$ und unter Vermeidung einer weiteren Insertion der scharfen Stahlinnenkanüle

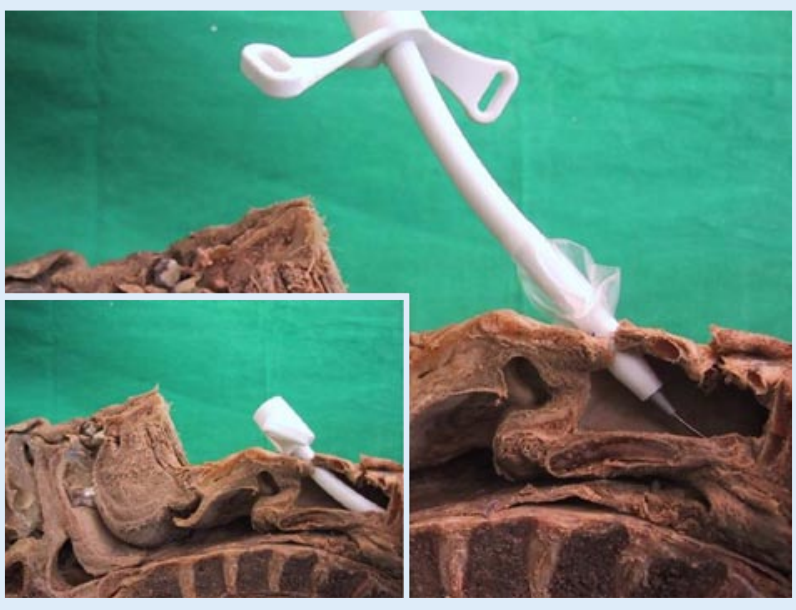

Abb. $13<$ Vorschieben von Dilatator und Koniotomiekanüle des MelkerSystems über den Führungsdraht in die Trachea. Darstellung am Sagittalschnitt des fixierten Leichenpräparates. Kleines Bild Lage in situ tenten im 2. Weiterbildungsjahr, benötigte in der gleichen Untersuchung sogar durchschnittlich 180 s (75-280 s) für die chirurgisch-anatomische Koniotomietechnik [23]. Die Bedeutung dieser Zeitdifferenz wird deutlich, wenn man in Rechnung stellt, dass der Patient, bei dem eine Koniotomie durchgeführt werden muss, während der Entscheidungsfindung bzw. während der frustranen Versuche einer Maskenbeatmung oder endotrachealen Intubation bereits hypoxisch sein kann. In der Untersuchung von Bair et al. [3] benötigten „emergency physicians“ und Flight nurses in 13 von 50 Fällen mehr als 2 min für die Durchführung der Koniotomie in chirurgischer bzw. Rapid-four-step-Technik. In einer aufgrund der kleinen Fall- und Anwenderzahl schwer zu interpretierenden aktuellen Untersuchung benötigten erfahrene Anwender für die chirurgisch-anatomische Technik an 5 unfixierten Leichen durchschnittlich 22,4 s (18-26 s). Trotz der Einschränkungen weist diese Untersuchung darauf hin, dass in der Hand des Geübten eine Koniotomie auch in chirurgisch-anatomischer Technik innerhalb von 30 s durchgeführt werden kann [7].

In einer weiteren Studie an unfixierten Leichen wurde die chirurgisch-anatomische Präparation mit der Seldinger-Technik verglichen. Dabei konnten für die primäre Erfolgsrate, für die Geschwindigkeit der Durchführung und für die resultierenden Läsionen benachbarter Gewebsstrukturen keine signifikanten Unterschiede gezeigt werden [14]. Als interessanter $\mathrm{Ne}$ benaspekt dieser Untersuchung wurde ei- 
ne Lernkurve bei der Durchführung der verschiedenen Verfahren beschrieben, und die Autoren kamen zu dem Schluss, dass jeder Arzt die Technik wählen sollte, die am ehesten den bisher durchgeführten manuellen Techniken entspricht: Während Anästhesisten und Intensivmediziner mit der Seldinger-Technik beispielsweise durch Anlegen von zentralvenösen Kathetern vertraut sind, haben überwiegend operativ tätige Kollegen vergleichsweise mehr Übung in chirurgischen Präparationstechniken. Die Autoren weisen aber auch darauf hin, dass der Seldinger-Draht bei nichtkorrekter Positionierung des „introducers" abknicken kann und dann für einen weiteren Punktionsversuch nicht mehr verwendbar ist.

\section{Qualität der Ventilation}

Eine andere aktuelle Untersuchung beschäftigte sich mit der Frage, wie gut über verschiedene Koniotomiekanülen ventiliert werden kann [12]. Die Beatmung einer Modelllunge unter Simulation verschiedener Atemwegswiderstände erfolgte dabei jeweils über einen Endotrachealtubus (ID $6 \mathrm{~mm}$ ), über nichtblockbare Koniotomiekanülen (ID 4 und $6 \mathrm{~mm}$ ) und über eine Jetventilationskanüle (13 G). In diesem Modell konnte bei der Beatmung über den Endotrachealtubus ein Atemminutenvolumen (AMV) von etwa $15 \mathrm{l} / \mathrm{min}$ erreicht werden. Das über die nichtblockbaren Koniotomiekanülen erreichte AMV war mit über $5 \mathrm{l} / \mathrm{min}$ deutlich geringer. Bei Beatmung über die 13-G-Kanüle mit ca. 2 l/min gelang keine adäquate Ventilation. Hierbei muss allerdings berücksichtigt werden, dass diese Untersuchungen nicht mit einem im Rettungsdienst üblicherweise eingesetzten (Hand-)Beatmungssystem, sondern mit einem konventionellen, im klinischen Routinebetrieb üblichen Narkosekreisteil durchgeführt wurden.

\section{Aspirationsschutz}

Für die Beurteilung des Aspirationsschutzes erscheint insbesondere von Bedeutung, ob die Koniotomiekanüle mit einem blockbaren Cuff versehen ist. Neben den bei der chirurgisch-anatomischen Technik eingesetzten Endotrachealtuben erscheint hier auch ein neuerdings mit einer block- baren Koniotomiekanüle (ID $5 \mathrm{~mm}$ ) angebotenes Koniotomieset (Melker Koniotomie-Set, Cook, Mönchengladbach) interessant zu sein.

\section{Komplikationen}

Eine unter Notfallbedingungen durchgeführte Koniotomie kann mit gravierenden Komplikationen behaftet sein. Insbesondere können Blutungen bei den chirurgisch-anatomischen Präpariertechniken die Sichtverhältnisse bei der Präparation und damit den Zugang zu den Atemwegen deutlich erschweren bzw. unmöglich machen. Bei eigenen Untersuchungen im Rahmen simulierter, in chirurgischer Präpariertechnik durchgeführten Notfallkoniotomien an insgesamt 38 Leichen kam es insbesondere zu venösen Gefäßverletzungen, Verletzungen des Schildknorpels und zur Tubusdeviation nach kranial (- Tabelle 4) [19, 20]. Die Tubusfehllagen wurden in adäquater Zeit erkannt und korrigiert; in allen Fällen wäre eine Beatmung möglich gewesen.

Bei 50 letztlich erfolgreichen, teilweise in konventioneller chirurgischer, teilweise in Rapid-four-step-Technik durchgeführten Notfallkoniotomien in einem US-amerikanischen Patientenkollektiv wurden als Komplikationen insbesondere prätracheale Tubusplatzierungen, die Notwendigkeit einer direkten Revision der Inzision und erforderliche Tubusrepositionen beschrieben ( $\bullet$ Tabelle 5) [3].

Auch dem Erfahrenen können nicht oder nur schwer beherrschbare Schwierigkeiten bei der Durchführung der Koniotomie begegnen. Insbesondere bei Patienten mit Struma kann es zu massiven Blutungen oder auch zu Schwierigkeiten beim Vorschieben der Koniotomiekanüle bzw. des Endotrachealtubus kommen, z. B. aufgrund einer Verlagerung oder Stenose der Trachea.

Eine besondere Situation kann sich bei zunehmender Schwellung im Halsbereich, wie beispielsweise beim Thoraxtrauma mit massivem Mediastinalemphysem, oder bei Verbrennungen ergeben. Aufgrund einer Umfangszunahme der Halsweichteile kann eine primär erfolgreich platzierte kurze Koniotomiekanüle sekundär dislozieren. In diesen Fällen sollte da-
Tabelle 4

Komplikationen bei Koniotomien in chirurgischer Präpariertechnik an der Leiche ( $n=38$; nach

Mutzbauer u. Georgieff [23] und Mutzbauer et al. [24])

\begin{tabular}{ll}
\hline Art der Komplikation & A \\
\hline Verletzung des Ringknorpels & 1 \\
\hline Fraktur des Ringknorpels & 2 \\
\hline Verletzung des Schildknorpels & 4 \\
\hline Verletzung des 1. Trachealrings & 1 \\
\hline $\begin{array}{l}\text { Verletzung des } \\
\text { Schilddrüsenisthmus }\end{array}$ & 1 \\
\hline $\begin{array}{l}\text { Verletzung des Lobus } \\
\text { pyramidalis }\end{array}$ & 2 \\
\hline
\end{tabular}

Venöse Gefäßverletzung 6

Arterielle Gefäßverletzung 2

Falscher Zugangsort $\quad 1$

(M. thyrohyoideus)

Paratracheale Tubuslage

Anzahl

(n)

1

2

4

1

1

2

Tubusdeviation nach kranial 1

Enger Abstand beider Knorpel

Ossifikation des

Lig. cricothyreoideum

Tabelle 5

Komplikationen bei Koniotomien $(n=50)$ an einem Traumazentrum mit angeschlossenem Luftrettungssystem. (Nach Bair et al. [3])

Art der Komplikation Anzahl

(n)

Falscher Zugangsort 1 (M. thyrohyroideus)

\begin{tabular}{lr}
\hline Hohe Tracheotomie & 1 \\
\hline Tubusplatzierung prätracheal & 8 \\
\hline $\begin{array}{l}\text { Direkte Revision der Inzision } \\
\text { erforderlich }\end{array}$ & 16 \\
\hline
\end{tabular}

Tubusrepositionierung 8 erforderlich

Übernahme durch 2. Behandler notwendig

Geringe Blutung

her die Verwendung eines Endotrachealtubus bevorzugt werden [22].

Studien, die Komplikationsraten verschiedener Methoden prospektiv evaluieren, liegen bisher nicht vor und erschei- 


$$
\frac{\text { Hier steht eine Anzeige }}{\text { This is an advertisement }}
$$

Springer 
nen auch zukünftig, insbesondere im präklinischen Bereich, nicht realisierbar. Es ist jedoch anzunehmen, dass aufgrund der geringeren Gewebetraumatisierung bei der Stahlinnenkanülentechnik weniger Blutungskomplikationen zu erwarten sind als bei der chirurgisch-anatomischen Präparation. Punktionsverfahren mit der Seldinger-Technik scheinen hier keine Vorteile zu besitzen, vermutlich deshalb, weil auch hier in der Regel eine Inzision mit dem Skalpell notwendig ist [11, 14].

Die Durchführung der Koniotomie in der propagierten konventionellen chirurgischen Präpariertechnik (s. oben) ist dadurch gekennzeichnet, dass der Notarzt seine Position am Kopf des Patienten zunächst aufgeben und sich (als Rechtshänder) auf die rechte Seite des Patienten und damit in eine im Rahmen des Atemwegsmanagements eher ungewohnte Position begeben muss [25]. Die Rapid-four-stepTechnik bietet hier möglicherweise Vorteile, da dieser Eingriff vom Kopfende des Patienten durchgeführt werden kann: Nach Querinzision von Haut und Lig. cricothyreoideum wird der Ringknorpel auf einen besonderen Trachealhaken aufgelagert und zusammen mit dem kaudalen Trachealanteil nach ventral gezogen; dann wird der Endotrachealtubus unterhalb des Hakens in die Trachea geschoben [9]. Zwei Studien an formalinfixierten Leichen verglichen die beiden Präparationsverfahren $[13,20]$. Die Rapid-four-step-Technik war schneller durchzuführen (im Mittel $43 \mathrm{~S}$ vs. $134 \mathrm{~s}$ ); es wurde aber auch eine höhere Komplikationsrate beschrieben. Eine abschließende Beurteilung zum Stellenwert dieses Verfahrens in der präklinischen Notfallmedizin ist zum gegenwärtigen Zeitpunkt noch nicht möglich.

\section{Ausbildungs- und Trainingsmöglichkeiten}

Die Punktionskoniotomie wird als Stahlinnenkanülentechnik oder Seldinger-Technik in einigen Zentren elektiv eingesetzt, einerseits zur Unterstützung der Oxygenierung, z. B. bei Patienten mit Oro- bzw. Hypopharynxtumoren, die ein konventionelles fiberoptisches Intubationsverfahren im Wachzustand nicht tolerieren würden [2], andererseits aber auch zum besseren $\mathrm{Ab}$ saugen von trachealem Sekret bei Patien- ten auf der Intensivstation, die nach der Extubation noch einen zu schwachen Hustenstoß aufweisen. Der Atemwegszugang mithilfe der Seldinger-Technik kann zudem im Rahmen von perkutanen Dilatationstracheotomien erlernt und bezüglich der Vorgehensweise dann auch auf die Koniotomie im Notfall transferiert werden. Darüber hinaus stehen Phantome verschiedener Anbieter zur Übung von Punktionstechniken zur Verfügung. Alle Koniotomietechniken, insbesondere die chirurgischen, können am Tiermodell oder am Leichenpräparat trainiert werden.

Bei der Interpretation entsprechender Studienergebnisse und zur Beantwortung der Frage, welches Verfahren für welche Anwender eher geeignet erscheint, muss allerdings berücksichtigt werden, dass auch diese Verfahren ausreichend Übung und Training zur erfolgreichen Anwendung erfordern. Wong et al. [29] konnten dabei nachweisen, dass unter Übungsbedingungen erst nach 5 in Seldinger-Technik durchgeführten Koniotomien diese Methode vom bisher unerfahrenen Anwender zeitnah und sicher durchgeführt werden kann. Die Autoren weisen allerdings einschränkend darauf hin, dass bei realen Notfallsituationen auftretende individuelle und situationsbedingte Probleme unter Studienbedingungen nicht ausreichend berücksichtigt werden können [29].

\section{Fazit für die Praxis}

Zentrale Argumente für die Verwendung blockbarer Endotrachealtuben und damit für die anatomisch-chirurgische Koniotomietechnik sind der damit verbundene sichere Aspirationsschutz und die Möglichkeit, eine Ventilation mit ausreichendem AMV durchführen zu können; dies ist beispielsweise bei der Versorgung SchädelHirn-traumatisierter Patienten essenziell. Bei Anwendung der meisten Punktionstechniken wird kein sicherer Aspirationsschutz gewährleistet. Die Möglichkeit einer adäquaten Ventilation ist nicht bei allen Systemen garantiert; lediglich eine ausreichende Oxygenierung kann bei reduziertem AMV unter Verwendung einer $\mathrm{F}_{1} \mathrm{O}_{2}$ von 1,0 sichergestellt werden. Welchem Koniotomieverfahren im Notfall der Vorzug gegeben wird, hängt letzlich von der Routine und Erfahrung des
Notarztes ab. Aufgrund des geringeren Zeitbedarfes kann die Punktionskoniotomie in der "Cannot-ventilate-cannot-intubate-Situation" auch zur "Überbrückung" sinnvoll sein, bis ein anderes Verfahren zur Atemwegssicherung, beispielsweise eine chirurgisch-anatomische Koniotomie, durchgeführt werden kann. Besonders für Notärzte aus nichtoperativen Fachdisziplinen empfiehlt sich der Besuch von praxisnahen Workshops, wie sie z. B. jährlich von der Klinik für Anästhesiologie in Zusammenarbeit mit dem Anatomischen Institut II der Universität Heidelberg, aber auch von anderen Institutionen angeboten werden. Hier kann die Koniotomie, neben anderen invasiven Notfalltechniken, am Leichenpräparat trainiert werden [30].

\section{Korrespondierender Autor}

Dr. med. Dr. med. dent. T. S. Mutzbauer

Klinik für Kiefer- und Gesichtschirurgie, Universitätsspital, Rämistrasse 100, 8091 Zürich, Schweiz

E-Mail: till.mutzbauer@usz.ch

\section{Danksagung}

Herrn Prof. Dr. M. Herrmann, Herrn Prof. Dr. J. Kirsch, Frau S. Doll und Herrn E. Voigt, Anatomisches Institut der Universität Ulm, wird an dieser Stelle nochmals für ihre außerordentliche Mühe und ihr Engagement bei der Bereitstellung anatomischer Präparate für unsere Untersuchungen und ihre Unterstützung bei der Anfertigung der zahlreichen Dokumentationsaufnahmen über viele Jahre gedankt.

Interessenkonflikt: Der korrespondierende Autor versichert, dass keine Verbindungen mit einer Firma, deren Produkt in dem Artikel genannt ist, oder einer Firma, die ein Konkurrenzprodukt vertreibt, bestehen.

\section{Literatur}

1. Agro F, Frass M, Benumof HL, Krafft P (2002) Current status of the Combitube ${ }^{\mathrm{TM}}$ : a review of the literature. J Clin Anesth 14:307-331

2. Ames W, Venn P (1998) Complication of the transtracheal catheter. Br J Anaesth 81:825

3. Bair AE, Panacek EA, Wisner DH, Bales R, Sakles JH (2003) Cricothyrotomy: a 5-year experience at one institution. J Emerg Med 24:151-156

4. Bossaert L (1999) Atemwegsmanagement. Notfall Rettungsmed 2:66-83

5. Brain Al (1983) The laryngeal mask - A new concept in airway management. Br J Anaesth 55:801805 
6. Braun U, Goldmann K, Hempel V, Krier C (2004) Airway management. Leitlinie der Deutschen Gesellschaft für Anästhesiologie und Intensivmedizin. Anaesthesiol Intensivmed 45:302-306

7. Breitmeier D, Schulz Y, Wilke N et al. (2004) Koniotomieübungen an der Leiche - Erfahrungen in der Ausbildung mit Medizinstudenten, Anästhesisten und Notärzten. Anasthesiol Intensivmed Notfallmed Schmerzther 39:94-100

8. Brimacombe J, Berry A (1995) The incidence of aspiration associated with the laryngeal mask airway: a meta-analysis of published literature. J Clin Anesth 7:297-305

9. Brofeldt BT, Panacek EA, Richards JR (1996) An easy cricothyrotomy approach: the rapid four-step technique. Acad Emerg Med 3:1060-1063

10. Caparosa RJ, Zavatsky AR (1957) Practical aspects of the cricothyroid space. Laryngoscope 67:577591

11. Chan TC, Vilke GM, Bramwell KJ, Davis DP, Hamilton RS, Rosen P (1999) Comparison of wire-guided cricothyrotomy versus standard surgical cricothyrotomy technique. J Emerg Med 17:957-962

12. Craven R, Vanner R (2004) Ventilation of a model lung using various cricothyrotomy devices. Anaesthesia 59:595-599

13. Davis D, Bramwell K, Vilke G et al. (1999) Cricothyrotomy technique: standard versus the rapid fourstep technique. J Emerg Med 17:17-21

14. Eisenburger P, Laczika K, List M et al. (2000) Comparison of conventional surgical versus Seldinger technique emergency cricothyrotomy performed by inexperienced clinicians. Anesthesiology 92:687-690

15. Feldmann H (1995) Die Behandlung akuter und chronischer Larynx- und Trachealstenosen im 19. und Anfang des 20. Jahrhunderts durch Tracheotomie, Koniotomie, Intubation und Dilation. Laryngorhinootologie 74:216-222

16. Frei FJ, Meier PYR, Lang FJW, Fasel JHD (1990) Krikothyreotomie mit dem „Quicktrach“-Koniotomiebesteck. Anasth Intensivther Notfallmed 25 [Suppl 1]:44-49

17. Gerich TG, Schmidt U, Hubrich V, Lobenhoffer HP, Tescherne H (1998) Prehospital airway management in the acutely injured patient: the role of surgical cricothyrotomy revisited. J Trauma 45:312314

18. Gerling MC, Davis DP, Hamilton RS, Morris GF, Vilke GM, Garfin SR, Hayden SR (2001) Effect of surgical cricothyrotomy on the unstable cervical spine in a cadaver model of intubation. J Emerg Med 20:1-5

19. Henderson JJ, Popat MT, Latto IP, Pearce AC (2004) Difficult Airway Society guidelines for management of the unanticipated difficult intubation. Anaesthesia 59:675-694

20. Holmes J, Panacek E, Sakles J, Brofeldt B (1998) Comparison of 2 cricothyrotomy techniques: standard method versus rapid 4-step technique. Ann Emerg Med 32:442-447

21. Keul W, Bernhard M, Völkl A, Gust R, Gries A (2004) Methoden des Atemwegsmanagement in der präklinischen Notfallmedizin. Anaesthesist 53:978992

22. Morris A, Lockey D, Coats T (1997) Fat necks: modification of a standard surgical airway protocol in the pre-hospital environment. Resuscitation 35:253254

23. Mutzbauer TS, Georgieff M (1996) How easy is emergency cricothyrotomy? Br J Anaesth 76 [Suppl 2]:116
24. Mutzbauer TS, Munz R, Helm M, Lampl LA, Herrmann M (2003) Notfallkoniotomie-Punktion oder chirurgisch-anatomische Präpariertechnik? Besonderheiten zweier Verfahren zur notfallmäßigen Atemwegssicherung dargestellt am anatomischen Präparat. Anaesthesist 52:304-310

25. Ott PM (2000) Der perakute Erstickungsnotfall: Was tun? Schweiz Rundsch Med Prax 89:571-574

26. Ravussin P, Freeman J (1985) A new transtracheal catheter for ventilation and resuscitation. Can Anesth Soc J 32:60-64

27. Thierbach A, Lipp M (1999) Fiberoptische Intubation im Notfall. Teil II - Evaluation in der Praxis. Notfall Rettungsmed 2:105-110

28. Wenzel V, Dörges V, Lindner KH (2001) Spontanatmung - Schnappatmung - Beatmung? Eine Analyse von verschiedenen Beatmungsstrategien be der kardiopulmonalen Reanimation. Notfall Rettungsmed 4:557-571

29. Wong DT, Prabhu AJ, Coloma M, Imasogie N, Chung FF (2003) What is the minimum training required for successful cricothyroidotomy ? A study in mannequins. Anesthesiology 98:349-353

30. ZinkW, Völkl A, Martin E, Gries A (2002) Die „INTECH-Studiengruppe": invasive Notfalltechniken (INTECH) - Ein Ausbildungskonzept in der Notfallmedizin? Anaesthesist 51:853-562

\section{BfArM schafft mehr Transparenz} Informationen über Risiken durch Medizinprodukte im Internet

Das Bundesinstitut für Arzneimittel und Medizinprodukte (BfArM) veröffentlicht seit Dezember 2004 aktuelle Informationen über Risiken durch Medizinprodukte und Empfehlungen zur Beseitigung, Verringerung oder Verhinderung des Wiederauftretens der Risiken. Die Informationen können im Verzeichnis "Medizinprodukte" der BfArM-Webseite (http://www.bfarm. de) eingesehen werden. Veröffentlicht werden Kundeninformationen von Herstellern über Rückrufe und sonstige korrektive Maßnahmen, Empfehlungen des BfArM und die Ergebnisse der von der Behörde durchgeführten wissenschaftlichen Aufarbeitung von Risikobewertungen. Die Informationen ergänzen die von Herstellern eigenverantwortlich durchgeführte Kundeninformation.

Die Veröffentlichungen auf der BfArMWebseite sollen dazu beitragen, etwaige Informationslücken zu schließen und das Problembewusstsein bei anderen Herstellern sowie Betreibern und Anwendern ähnlicher Medizinprodukte zu erhöhen.

Quelle: Bundesinstitut für Arzneimittel und Medizinprodukte (http://www.bfarm.de)

\section{Personalia}

Herr Prof. Dr. med. Bernd W. Böttiger, Geschäftsführender Oberarzt der Klinik für Anaesthesiologie am Universitätsklinikum Heidelberg wurde zum "Chairman Elect" des European Resuscitation Council (ERC) gewählt. 